\title{
Tumor DNA Sequencing
}

National Cancer Institute ( $\mathrm{NCl})$

\section{Source}

National Cancer Institute (NCI). Tumor DNA Sequencing.

Sequencing of somatic tissue, such as tumors, refers to looking for variants in DNA that typically occur after conception. Somatic mutations can occur in any of the cells of the body except the germ cells (sperm and egg) and therefore are not passed on to children. These variants can (but do not always) cause cancer or other diseases. 